\title{
Spin Polarized Current Generation from Quantum Dots without Magnetic Fields
}

\section{Citation}

Krich, Jacob J. and Bertrand I. Halperin. 2008. Spin polarized current generation from quantum dots without magnetic fields. Physical Review B 78, no. 3: 035338.

\section{Published Version}

http://dx.doi.org/10.1103/PhysRevB.78.035338

\section{Permanent link}

http://nrs.harvard.edu/urn-3:HUL.InstRepos:3110943

\section{Terms of Use}

This article was downloaded from Harvard University's DASH repository, and is made available under the terms and conditions applicable to Open Access Policy Articles, as set forth at http:// nrs.harvard.edu/urn-3:HUL.InstRepos:dash.current.terms-of-use\#OAP

\section{Share Your Story}

The Harvard community has made this article openly available.

Please share how this access benefits you. Submit a story.

\section{Accessibility}




\title{
Spin-polarized current generation from quantum dots without magnetic fields
}

\author{
Jacob J. Krich and Bertrand I. Halperin \\ Physics Department, Harvard University, Cambridge, Massachusetts 02138, USA \\ (Received 16 January 2008; revised manuscript received 8 June 2008; published 31 July 2008)
}

\begin{abstract}
An unpolarized charge current passing through a chaotic quantum dot with spin-orbit coupling can produce a spin-polarized exit current without magnetic fields or ferromagnets. We use random matrix theory to estimate the typical spin polarization as a function of the number of channels in each lead in the limit of large spin-orbit coupling. We find rms spin polarizations up to $45 \%$ with one input channel and two output channels. Finite temperature and dephasing both suppress the effect, and we include dephasing effects using a variation of the third lead model. If there is only one channel in the output lead, no spin polarization can be produced, but we show that dephasing lifts this restriction.
\end{abstract}

DOI: 10.1103/PhysRevB.78.035338

PACS number(s): 72.25.-b, 73.63.Kv, 75.47. $-\mathrm{m}$, 85.75. $-\mathrm{d}$

\section{INTRODUCTION}

The generation and control of spin-polarized currents, in particular without magnetic fields or ferromagnets, is a major focus of recent experimental and theoretical work. This includes the spin Hall effect, which produces spin currents transverse to an electric field in a two-dimensional electron system (2DES) with spin-orbit coupling, with spin accumulation at the edges. ${ }^{1}$ Similarly, the magnetoelectric effect ${ }^{2-4}$ produces a steady-state spin accumulation when an electric field is applied to a $2 \mathrm{DES}$ with spin-orbit coupling. The accumulation can be uniform ${ }^{5,6}$ in the case of uniform Rashba spin-orbit coupling ${ }^{7}$ or at the edges of a channel in either the Rashba model ${ }^{8-10}$ or with spin-orbit coupling induced by lateral confinement. ${ }^{11,12}$ Experiments have observed current induced spin polarization in $n$-type three-dimensional (3D) samples $^{13}$ and in two-dimensional (2D) hole systems ${ }^{14,15}$ with spin polarization estimated to be up to $10 \% .{ }^{15}$ Further work suggests that a spin-polarized current can be produced in quantum wire junctions, ${ }^{16,17}$ by a quantum point contact (QPC), ${ }^{18,19}$ in a carbon nanotube, ${ }^{20}$ in a ballistic ratchet, ${ }^{21}$ in a torsional oscillator, ${ }^{22}$ in vertical transport through a quantum well, ${ }^{23}$ or in disordered mesoscopic systems. ${ }^{24}$

Here we show that generating a polarized current from an unpolarized current is a generic property of scattering through a mesoscopic system with spin-orbit coupling. We propose using many-electron quantum dots (outside the Coulomb blockade regime) with spin-orbit coupling to produce partially spin-polarized currents without magnetic fields or ferromagnets. Due to the complicated boundary conditions of the quantum dot, we do not solve for the spin polarization in terms of any particular spin-orbit coupling model, geometry, and contact configuration. We estimate the effect for a ballistic system in the limit of strong spin-orbit coupling by performing a random matrix theory (RMT) calculation for the spin polarization, allowing consideration of realistic quantum dot devices robust to details of shape and contact placement. Finely tuned systems should be able to exceed these polarizations, but these results provide a useful benchmark for whether a particular tuned system is better than a generic chaotic one. We use a density-matrix formalism throughout, which allows us to develop straightforwardly a spin-conserving dephasing probe, using a variant of the third lead technique for accounting for dephasing. Dephasing and finite temperature both reduce the expected polarization. Without dephasing, we find that if there is only one outgoing channel then no spin polarization is possible, which was first shown simultaneously by Zhai and $\mathrm{Xu}^{25}$ and Kiselev and Kim. ${ }^{26}$ Interestingly, with dephasing, spin polarization can be produced with only one outgoing channel. The case of polarized input currents will be discussed elsewhere. ${ }^{27}$ Analogous calculations have been performed by Bardarson et al. ${ }^{28}$ in a four-terminal geometry to study the transverse spin current produced by an applied charge current.

\section{SETUP AND SYMMETRY RESTRICTIONS}

We consider noninteracting electrons in a quantum dot with two attached leads connected to large reservoirs. For any electron current entering from the leads, we can describe the output state in the leads in terms of the $S$ matrix of the dot, including any tunnel barriers between the leads and the dot. We assume negligible spin-orbit coupling in the leads and consider the lead on the left (right) to have $N(M)$ spindegenerate channels at least partially open at the Fermi energy and let $K=N+M$. As usual, the channel wave functions are normalized so all channels have the same flux. The $S$-matrix $S$ is a $2 K \times 2 K$ unitary matrix of complex numbers. For spin $1 / 2$ particles with spin-orbit coupling, however, it is convenient to consider $S$ to be a $K \times K$ matrix of $2 \times 2$ matrices. We represent these $2 \times 2$ matrices using quaternions, where a quaternion $q=q^{(0)} 1_{2}+i \Sigma_{\mu=1}^{3} q^{(\mu)} \sigma_{\mu}$, where $\sigma_{\mu}$ are the Pauli matrices and $q^{(\mu)} \in \mathrm{C}$. We give a brief introduction to quaternions in Appendix A. The quaternion representation is convenient, as the time-reversal operation for a scattering matrix can simply be written as $S \rightarrow S^{R}$, where $R$ gives the quaternion dual [which takes the transpose and sends $q^{(1,2,3)} \rightarrow-q^{(1,2,3)}$ (see Appendix A).$^{29}$ The $S$ matrix of a system with time-reversal symmetry (TRS) is self-dual.

If $w^{\text {in }}$ ( $\left.w^{\text {out }}\right)$ is the $K \times K$ quaternion density matrix of the incoming (outgoing) current, $w^{\text {out }}=S w^{\text {in }} S^{\dagger}$. The density matrix describing the unpolarized incoherent combination of all $N$ incoming channels is

$$
w^{\text {in }}=\frac{1}{2 N}\left(\begin{array}{ll}
\mathbb{1}_{N} & \\
& 0_{M}
\end{array}\right) .
$$

That is, $w^{\text {in }}=P_{L} / 2 N$, where $P_{L}$ is the projection onto the channels of the left lead. We choose $\operatorname{tr} w^{\text {in }}=1 / 2$ due to the 
quaternion trace convention (see Appendix A), so $w^{\text {in }}$ represents one incident electron.

The Landauer-Büttiker formula gives the conductance in terms of the $S$ matrix. ${ }^{30}$ We write the $K \times K$ quaternion $S$ matrix as

$$
S=\left(\begin{array}{ll}
r & t^{\prime} \\
t & r^{\prime}
\end{array}\right),
$$

with $r\left(r^{\prime}\right)$ being the $N \times N(M \times M)$ reflection matrix and $t$ $\left(t^{\prime}\right)$ the $M \times N(N \times M)$ transmission matrix. Then we write the Landauer-Büttiker formula in units of $2 e^{2} / h$ as

$$
G=\operatorname{tr}\left(t t^{\dagger}\right)=\operatorname{tr}\left(P_{R} S P_{L} S^{\dagger}\right)=2 N \operatorname{tr}\left(P_{R} S w^{\text {in }} S^{\dagger}\right)=2 N \operatorname{tr}\left(P_{R} w^{\text {out }}\right),
$$

where $P_{R}$ is the projection onto the channels of the right lead. Since $w^{\text {in }}$ is normalized to represent one input particle entering the system, $g=2 \operatorname{tr}\left(P_{R} w^{\text {out }}\right)$ is the probability for that particle to exit through the right lead. The conductance is $N$ times this probability, so we call $g$ the conductance per channel in the left lead.

Similarly, we define a vector spin conductance ${ }^{25}$ (i.e., exit spin current divided by voltage) $\vec{G}^{s}$ in units of $e / 2 \pi$ as

$$
\vec{G}^{s}=\operatorname{tr}\left(\vec{\sigma} t t^{\dagger}\right)=2 N \operatorname{tr}\left(\vec{\sigma} P_{R} w^{\text {out }}\right) .
$$

Then

$$
\overrightarrow{g^{s}}=2 \operatorname{tr}\left(\vec{\sigma} P_{R} w^{\text {out }}\right)
$$

is the spin conductance per channel in the left lead. Hence, $g_{\mu}^{s}$ is the $\mu$ component of the spin polarization of the exit current times the probability of exiting into the right lead. Thus, the spin polarization of the current in the right lead is $\vec{p}=\vec{g}^{s} / g$, with $|p| \leq 1$.

We can, of course, construct $g, \overrightarrow{g^{s}}$, and $\vec{p}$ using only the $S$ matrix and not the density matrices $w^{\text {in }}$ and $w^{\text {out }}$. The densitymatrix approach, however, gives the flexibility to consider arbitrarily correlated states of incoming current and also to look for arbitrary correlations in the outgoing current. ${ }^{27} \mathrm{We}$ will also use it to straightforwardly derive a method of accounting for nonmagnetic dephasing in a device with spinorbit coupling. To complete the translation to the standard notation of conductances, we consider sending up- or downpolarized electrons into a sample and collecting either up- or down-polarized electrons, giving a conductance matrix ${ }^{31}$

$$
\mathbf{G}=\left(\begin{array}{ll}
G_{\uparrow \uparrow} & G_{\uparrow \downarrow} \\
G_{\downarrow \uparrow} & G_{\downarrow \downarrow}
\end{array}\right),
$$

with the total charge conductance being $G=G_{\uparrow \uparrow}+G_{\uparrow \downarrow}+G_{\downarrow \uparrow}$ $+G_{\downarrow \downarrow} . G_{\sigma, \sigma^{\prime}}$ is the conductance for an input current of spin $\sigma^{\prime}$ and an exit current of spin $\sigma$ for $\sigma, \sigma^{\prime}=\uparrow, \downarrow$. We translate the quaternion representation into the standard notation by noting that the up-polarized incoherent input current has input density matrix $w_{\uparrow}^{\text {in }}=\frac{1+\sigma_{3}}{2 N} P_{L}$. The output density matrix is $w_{\uparrow}^{\text {out }}=S w_{\uparrow}^{\text {in }} S^{\dagger}$ and the portion representing the output in the right lead is $t \frac{1+\sigma_{3}}{2 N} t^{\dagger}$. The Landauer-Büttiker formula gives, in units of $2 e^{2} / h$,

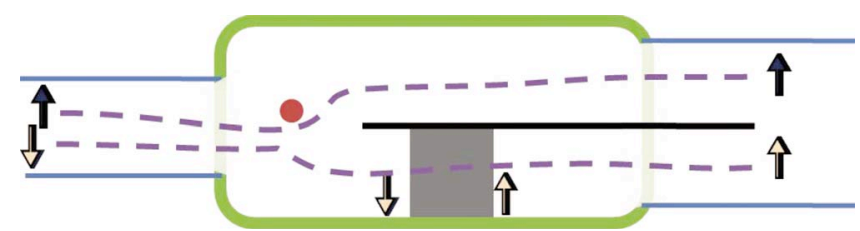

FIG. 1. (Color online) A special model quantum dot with $N=1$ channels in the left lead and $M=2$ channels in the right lead. A skew scatterer sends all $\hat{z}$ spins to the top channel and all $-\hat{z}$ spins to the bottom channel. The shaded area in the bottom channel has Rashba spin-orbit coupling of precisely the strength to rotate a down spin at the Fermi energy to an up spin, thus producing a perfectly spin-polarized exit current from any input current, while respecting time-reversal symmetry.

$$
G_{\uparrow \uparrow}=N \operatorname{tr}\left(P_{R} \frac{1+\sigma_{3}}{2} w_{\uparrow}^{\text {out }}\right)=\operatorname{tr}\left(\frac{1+\sigma_{3}}{2} t \frac{1+\sigma_{3}}{2} t^{\dagger}\right) .
$$

Similarly,

$$
\begin{aligned}
& G_{\downarrow \uparrow}=\operatorname{tr}\left(\frac{1-\sigma_{3}}{2} t \frac{1+\sigma_{3}}{2} t^{\dagger}\right), \\
& G_{\uparrow \downarrow}=\operatorname{tr}\left(\frac{1+\sigma_{3}}{2} t \frac{1-\sigma_{3}}{2} t^{\dagger}\right), \\
& G_{\downarrow \downarrow}=\operatorname{tr}\left(\frac{1-\sigma_{3}}{2} t \frac{1-\sigma_{3}}{2} t^{\dagger}\right),
\end{aligned}
$$

from which we see that $G=\operatorname{tr}\left(t t^{\dagger}\right)$, which is the usual Landauer-Büttiker formula. ${ }^{30}$

Though there are several proposed spin-orbit coupled systems that demonstrate spin polarization from unpolarized input, in many cases the effect is subtle. ${ }^{18,19,21,32,33}$ Here we give a simple, idealized thought experiment to show that time-reversal symmetry does not forbid generating a spinpolarized current from an unpolarized input current. Consider a system with $N=1$ and $M=2$, as illustrated in Fig. 1 . All input electrons are incident on a perfect skew scatterer, ${ }^{1}$ which sends spins quantized in the $+z$ direction into exit channel 1 and spins quantized in the $-z$ direction into exit channel 2. Exit channel 2 has a region with Rashba spin-orbit coupling ${ }^{7}$ which is precisely of the strength and length necessary to rotate $-z$ spins to $+z$. Thus, all spins incident from the left lead exit with their spins up, and the system respects TRS, since skew scattering and Rashba spin-orbit interaction are each time-reversal symmetric.

We illustrate by constructing $S$ explicitly. We can express the scattering matrix for this thought experiment (up to an overall phase) in the $6 \times 6$ and $3 \times 3$ representations as

$$
S=\left(\begin{array}{cccccc}
0 & 0 & 0 & 0 & 0 & -1 \\
0 & 0 & 0 & 1 & 0 & 0 \\
1 & 0 & 0 & 0 & 0 & 0 \\
0 & 0 & 0 & 0 & e^{i \theta} & 0 \\
0 & 1 & 0 & 0 & 0 & 0 \\
0 & 0 & -e^{i \theta} & 0 & 0 & 0
\end{array}\right),
$$




$$
\equiv \frac{1}{2}\left(\begin{array}{ccc}
0 & 1-\sigma_{z} & -\sigma_{x}-i \sigma_{y} \\
1+\sigma_{z} & 0 & e^{i \theta}\left(\sigma_{x}-i \sigma_{y}\right) \\
\sigma_{x}+i \sigma_{y} & -e^{i \theta}\left(\sigma_{x}-i \sigma_{y}\right) & 0
\end{array}\right),
$$

where $\theta \in[0,2 \pi)$ and $r$ and $t$ have been determined by the above description, while the rest of the matrix is given by TRS and unitarity. The unpolarized input quaternion density matrix is

$$
w^{\text {in }}=\left(\begin{array}{ccc}
1 / 2 & & \\
& 0 & \\
& & 0
\end{array}\right),
$$

giving

$$
w^{\text {out }}=S w^{\text {in }} S^{\dagger}=\frac{1}{4}\left(\begin{array}{ccc}
0 & 0 & 0 \\
0 & 1+\sigma_{z} & 0 \\
0 & 0 & 1+\sigma_{z}
\end{array}\right),
$$

so Eq. (4) gives $\overrightarrow{g^{s}}=\vec{p}=\hat{z}$, as stated above.

We now prove that having at least two channels in the outgoing lead is essential. That is, for a dot with TRS and $K$ channels in attached leads, if an unpolarized equally weighted incoherent current is sent into $N=K-1$ of the channels, then the spin polarization in the remaining channel must be zero. This result has been shown before, ${ }^{25,26}$ but the quaternion formalism with density matrices makes it particularly transparent, so we include the proof here.

We start with

$$
w^{\text {in }}=\frac{1}{2 N}\left(\begin{array}{cc}
\mathbb{1}_{N} & \\
& 0
\end{array}\right)=\frac{\mathbb{1}_{K}-P_{K}}{2 N},
$$

where $P_{K}$ is the projection onto the $K$ th channel. The quaternion scattering matrix satisfies $S=S^{R}$ since TRS is unbroken, and

$$
w^{\text {out }}=\frac{S S^{\dagger}-S P_{K} S^{\dagger}}{2 N}=\frac{1_{K}-S P_{K} S^{\dagger}}{2 N} .
$$

Note that $S=S^{R}$ implies both $S^{\dagger}=S^{*}$ and $S_{i i} \in \mathrm{C}$ for $i$ $=1, \ldots, K$ [see Eq. (A3)].

Using Eq. (4), the spin conductance is $g_{\mu}^{s}=2 i\left[w_{K K}^{\text {out }}\right]^{(\mu)}$, where $[q]^{(\mu)}$ is the $\mu$ component of the quaternion $q$. In particular, if $w_{K K}^{\text {out }}$ has no quaternion part, then $g_{\mu}^{s}=0$. We have

$$
w_{K K}^{\text {out }}=\frac{1-S_{K K} S_{K K}^{*}}{2 N},
$$

and $S_{K K} S_{K K}^{*}$ is real, so $w_{K K}^{\text {out }} \in \mathbb{R}$ and $\overrightarrow{g^{s}}=0$. This proof applies with channels that are fully open or have tunnel barriers, as it requires only that the $S$ matrix satisfies TRS and unitarity, which are unchanged by tunnel barriers. We note further that if $K>2$ then (1) the reflected current in any of the $K-1$ input channels can be spin polarized and (2) if the input current goes through less than $K-1$ channels, then the remaining channels can have a spin polarization, as shown in the example of Fig. 1.

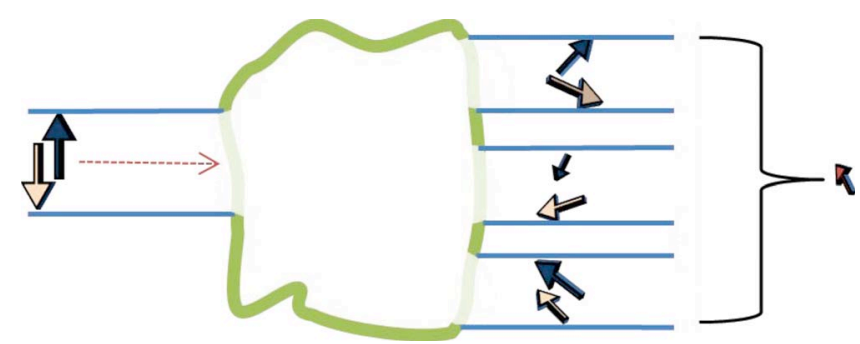

FIG. 2. (Color online) Generic device with one input channel. Unpolarized current, consisting of equal parts spin-up and spindown components, is incident on a chaotic quantum dot from a single channel on the left. The irregularly shaped region in the middle is a quantum dot in the strong spin-orbit regime. The spin-up incident current has a probability to exit into each of the three channels of the right lead with a different spin direction in each channel, indicated by the direction of the dark arrows. Similarly for the spin-down incident current, indicated by the light arrows. The exit channels are shown spatially separated, for convenience. There is also a probability to scatter back into the left lead (not shown). The total spin current in the exit lead is given by summing the probabilities and directions of the six spin polarizations shown, which is the meaning of Eq. (4) and is indicated by the arrow on the right.

\section{RANDOM MATRIX THEORY}

The device illustrated in Fig. 1 cannot easily be made, but realistic devices with spin-orbit coupling will show a similar (albeit weaker) spin current generation. An intuitive picture for the generation of spin currents from an applied voltage in a realistic device is to consider the case with $N=1$ and $M>1$. A current of spin-up electrons incident from the left has some probability to exit in each of the $M$ channels of the right lead, each associated with a spin-polarization direction; in the strong spin-orbit limit, these spin polarizations can have arbitrary directions. The same is true for a current of spin-down electrons incident from the left. As illustrated in Fig. 2, a charge current entering from the left is a combination of these spin-up and spin-down currents. The spin polarization of the current in the right lead is given by the sum of the spin polarizations of each of the $2 M$ currents in the channels of the right lead, weighted by the probability of the particle to enter that channel. Despite the presence of timereversal symmetry, there is no requirement that this polarization sums to zero, as seen explicitly in the example of Fig. 1. In the chaotic strong spin-orbit limit, these $2 M$ vectors are (almost) uncorrelated, and their sum will generically be nonzero. As $M$ or $N$ are increased, however, there are more independent vectors contributing to the sum, which generally brings the sum closer to zero. We formalize this intuition using random matrix theory.

We estimate the expected spin polarization in realistic situations by using random scattering matrix theory. We assume that the mean dwell time $\tau_{d}=2 \pi \hbar / K \Delta$ of particles in the dot is much greater than the Thouless time $\tau_{\mathrm{Th}}=L_{d} / v_{F}$, where $K$ is the number of fully open orbital channels attached to the dot, $\Delta=2 \pi \hbar^{2} / m A$ is the mean orbital level spacing, $m$ is the effective mass, $A$ is the area of the dot, $v_{F}$ is the Fermi velocity, and $L_{d}$ is a typical length scale of the 
quantum dot. We further assume the strong spin-orbit limit, where the spin-orbit time $\tau_{\text {so }}$ is much less than $\tau_{d}$. Since $\tau_{d}$ $=m A / \hbar K$, for a sufficiently large $A$, even a material with "weak" spin-orbit coupling will be in the strong spin-orbit limit. The crossover from weak to strong spin-orbit coupling in chaotic quantum dots has been studied in the $K \gg 1$ limit in the context of adiabatic spin pumping. ${ }^{34}$

For dots with strong spin-orbit coupling, we assume that the $S$ matrix is chosen from the uniform distribution of unitary matrices subject to TRS, called the circular symplectic ensemble (CSE). ${ }^{29,35}$ We find the root-mean-square (rms) magnitude of the spin conductance on averaging over the CSE, which gives the typical spin conductance magnitude to be expected from chaotic devices. Such an averaging can be realized in practice by small alterations of the dot shape. ${ }^{36,37}$

By symmetry, $\left\langle g_{\mu}^{s}\right\rangle=0$ for $\mu=1,2,3$. Using Eq. (4), we evaluate

$$
\left\langle\left(g^{s}\right)^{2}\right\rangle=4\left\langle\operatorname{tr}\left(\sigma_{\mu} P_{R} S w^{\text {in }} S^{\dagger}\right) \operatorname{tr}\left(\sigma_{\mu} P_{R} S w^{\text {in }} S^{\dagger}\right)\right\rangle,
$$

where we sum over $\mu$.

We use the technique for averaging over the CSE described by Brouwer and Beenakker in Sec. V of Ref. 38. We just need two generic averages, which we will use repeatedly. The first is of the form $\left\langle F_{1}(S)\right\rangle=\left\langle\operatorname{tr}\left(A S B S^{\dagger}\right)\right\rangle$, where $A$ and $B$ are constant $K \times K$ quaternion matrices and the average is taken over $S$ chosen from the CSE of $K \times K$ quaternion self-dual matrices. Then ${ }^{38}$

$$
\left\langle F_{1}\right\rangle=\frac{1}{2 K-1}\left[2 \operatorname{tr}(A) \operatorname{tr}(B)-\operatorname{tr}\left(A B^{R}\right)\right] .
$$

The second average we need is $\left\langle F_{2}(S)\right\rangle$ $=\left\langle\operatorname{tr}\left(A S B S^{\dagger}\right) \operatorname{tr}\left(C S D S^{\dagger}\right)\right\rangle$, where $A, B, C$, and $D$ are constant $K \times K$ quaternion matrices and $A B=A D=C B=C D=0$. We find $^{38}$

$$
\begin{aligned}
\left\langle F_{2}\right\rangle= & \frac{1}{\Lambda}\{(K-1)[4 \operatorname{tr} A \operatorname{tr} B \operatorname{tr} C \operatorname{tr} D+\operatorname{tr}(A C) \operatorname{tr}(B D)] \\
& -[\operatorname{tr} A \operatorname{tr} C \operatorname{tr}(B D)+\operatorname{tr}(A C) \operatorname{tr} B \operatorname{tr} D]\},
\end{aligned}
$$

where $\Lambda=K(2 K-1)(2 K-3)$.

Using Eq. (18), we find

$$
\left\langle\left(g^{s}\right)^{2}\right\rangle=3 \frac{M(M-1)}{N \Lambda},
$$

where we used $\operatorname{tr}\left(\sigma_{\mu} P_{R}\right)=0, \operatorname{tr}\left(P_{R}^{2}\right)=M, \operatorname{tr} w^{\text {in }}=1 / 2$, and $\operatorname{tr}\left[\left(w^{\text {in }}\right)^{2}\right]=1 / 4 N$. Note that when $M=1,\left\langle\left(g^{s}\right)^{2}\right\rangle=0$, consistent with the general symmetry.

If we are interested in the mean-square polarization of the exit current, $\left\langle p^{2}\right\rangle=\left\langle\left(g^{s}\right)^{2} g^{-2}\right\rangle$, we can approximate it by $\left\langle\left(g^{s}\right)^{2}\right\rangle /\langle g\rangle^{2}$. This approximate form is useful for analytical progress and will be compared with numerical results. Using Eq. (17),

$$
\langle g\rangle=\frac{2 M}{2 K-1},
$$

which, combined with Eq. (19), gives
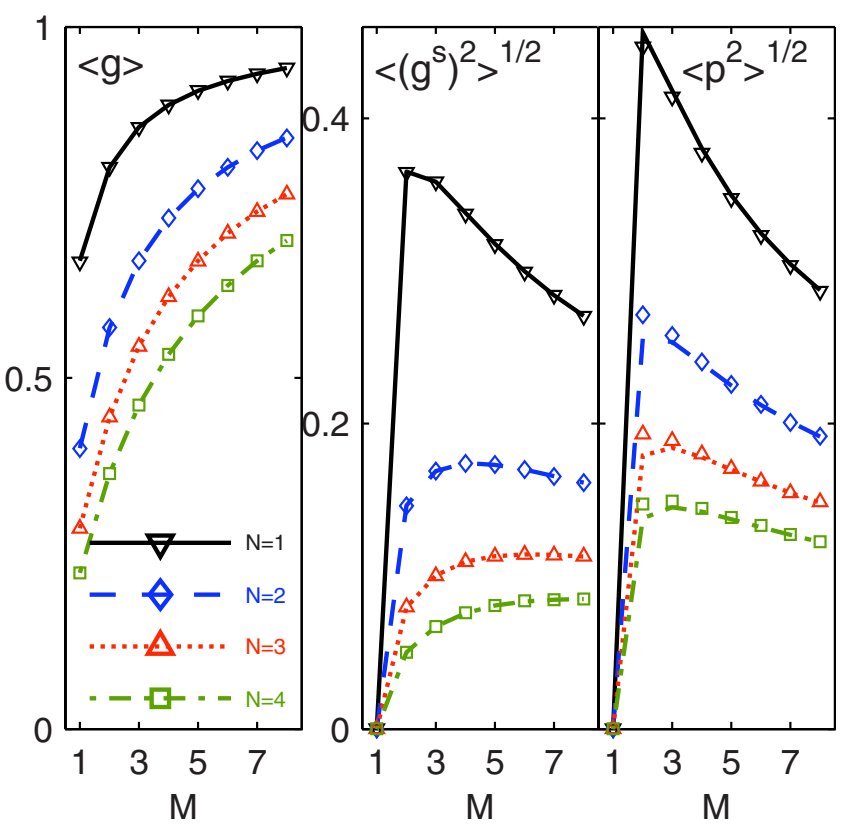

FIG. 3. (Color online) Numerical (symbols) and analytical (lines) results for normalized mean conductance $\langle g\rangle$, rms spin conductance $g^{s}$, and rms spin polarization $p$ of current exiting a chaotic quantum dot with $N(M)$ channels in the entrance (exit) lead. An average over $60000 S$ matrices from the CSE was performed for each data point. The lines are from Eqs. (19)-(21).

$$
\left\langle p^{2}\right\rangle \approx \frac{3(M-1)(2 K-1)}{4 M N K(2 K-3)} .
$$

We study the approximation $\left\langle\left(g^{s}\right)^{2} g^{-2}\right\rangle \approx\left\langle\left(g^{s}\right)^{2}\right\rangle /\langle g\rangle^{2}$ numerically. We choose a $2 K \times 2 K$ complex Hermitian matrix from the Gaussian unitary ensemble ${ }^{29}$ and find the unitary matrix $U$ which diagonalizes it. We multiply columns of $U$ by random phases, map $U$ into a $K \times K$ matrix of quaternions, and construct unitary self-dual $S$ by setting $S=U U^{R}$, giving $S$ chosen from the CSE. ${ }^{39}$

Figure 3 shows the numerical and analytical results, which agree quantitatively for $\left\langle g^{2}\right\rangle$ and $\left\langle\left(g^{s}\right)^{2}\right\rangle$ and qualitatively for $\left\langle p^{2}\right\rangle$. The largest percentage disagreement for $\left\langle p^{2}\right\rangle$ is $7 \%$.

\section{DEPHASING}

We add dephasing to this setup using the dephasing voltage probe technique. ${ }^{40-42} \mathrm{We}$ add a fictitious voltage probe drawing no current with $N_{\phi}=2 \pi \hbar / \Delta \tau_{\phi}$ fully open orbital channels, so the time for escape into the dephasing lead is the dephasing time $\tau_{\phi}$. If the dephasing process is spin independent, then it is appropriate to conserve the spin of the reinjected electrons, and we extend the third lead model to allow this. If, however, dephasing processes relax the spin, then it is appropriate for the dephasing lead to reinject unpolarized current, preserving only electron number. We consider both of these models, with emphasis on the first, as it is new in this work.

In our formulation, we explicitly model reinjection of electrons from the fictitious voltage lead by modifying $w^{\text {in }}$ to 
include incoherent reinjection from the dephasing lead. In either model of dephasing, the reinjection matches the total charge current absorbed by the dephasing lead but distributes the charge current evenly between the channels and removes the correlations. In the spin-conserving case, the reinjection also preserves the spin current. Consider $\eta_{\mu}$ $=\operatorname{tr}\left(\sigma_{\mu} P_{\phi} S w_{0}^{\text {in }} S^{\dagger}\right)$, where $\mu=0,1,2,3, \sigma_{0}=\mathbb{1}_{2}, P_{\phi}$ is the projection operator onto the dephasing lead's channels, and $w_{0}^{\text {in }}$ is the input density matrix. Then $2 \eta_{0}$ is the probability for a particle to enter the dephasing lead, and $2 \vec{\eta}$ is the spin conductance into the dephasing lead, which is proportional to the spin current into the dephasing lead.

We reinject from the dephasing lead with

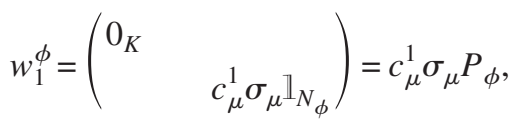

where we sum over repeated index $\mu$. To preserve both spin and charge currents, we set $c_{\mu}^{1}=\eta_{\mu} / N_{\phi}$. Some of this reinjected current reflects back into the dephasing lead, so it must be reinjected again. We define a $4 \times 4$ complex matrix $\Xi_{\mu \nu}=\operatorname{tr}\left(\sigma_{\nu} P_{\phi} S \sigma_{\mu} P_{\phi} S^{\dagger}\right)$, which gives the charge/spin current in the dephasing lead due to this reinjection. Defining $w^{\text {in }}$ $=w_{0}^{\text {in }}+w_{\phi}^{\text {in }}$, this procedure gives

$$
\begin{aligned}
w_{\phi}^{\text {in }} & =\sum_{n=1}^{\infty} w_{n}^{\phi}=P_{\phi} \sigma_{\mu} \operatorname{tr}\left(\sigma_{\nu} P_{\phi} S w_{0}^{\text {in }} S^{\dagger}\right) \sum_{n=1}^{\infty} \frac{\left(\Xi^{n-1}\right)_{\mu \nu}}{N_{\phi}^{n}} \\
& =P_{\phi} \sigma_{\mu} \operatorname{tr}\left(\sigma_{\nu} P_{\phi} S w_{0}^{\text {in }} S^{\dagger}\right)\left(N_{\phi} \delta_{\mu \nu}-\Xi_{\mu \nu}\right)^{-1},
\end{aligned}
$$

where we sum over repeated indices $\mu, \nu=0,1,2,3$. This result holds for any input current, not just the unpolarized incoherent $w_{0}^{\text {in }}$ discussed here.

We approximate $w_{\phi}^{\text {in }}$ by replacing $\Xi_{\mu \nu}$ with its average in Eq. (23), similar to Eq. (21). Using Eq. (17),

$$
\left\langle\Xi_{\mu \nu}\right\rangle=\frac{N_{\phi}}{2 K_{\phi}-1}\left[2\left(N_{\phi}-1\right) \delta_{\mu 0} \delta_{\nu 0}+\delta_{\mu \nu}\right],
$$

where $K_{\phi}=N+M+N_{\phi}$. We further replace $\operatorname{tr}\left(\sigma_{\nu} P_{\phi} S w_{0}^{\text {in }} S^{\dagger}\right)$ by its average,

$$
\left\langle\operatorname{tr}\left(\sigma_{\nu} P_{\phi} S w_{0}^{\mathrm{in}} S^{\dagger}\right)\right\rangle=\frac{\delta_{\nu 0} N_{\phi}}{2 K_{\phi}-1},
$$

which gives

$$
w^{\text {in }} \approx w_{0}^{\text {in }}+P_{\phi} / 2 K .
$$

Note that Eq. (26) satisfies unitarity only on average; the total probability of exiting either through the right or left lead equals 1 only on average.

In the case of a spin-relaxing dephasing lead, Eq. (23) becomes

$$
w_{\phi}^{\text {in }}=P_{\phi} \frac{\operatorname{tr}\left(P_{\phi} S w_{0}^{\text {in }} S^{\dagger}\right)}{N_{\phi}-\operatorname{tr}\left(P_{\phi} S P_{\phi} S^{\dagger}\right)} .
$$

Interestingly, approximating by taking averages separately of the numerator and denominator gives the same answer as in Eq. (26).

As is clear from Eq. (26), the approximations of Eqs. (24) and (25) result in unpolarized reinjection from the dephasing

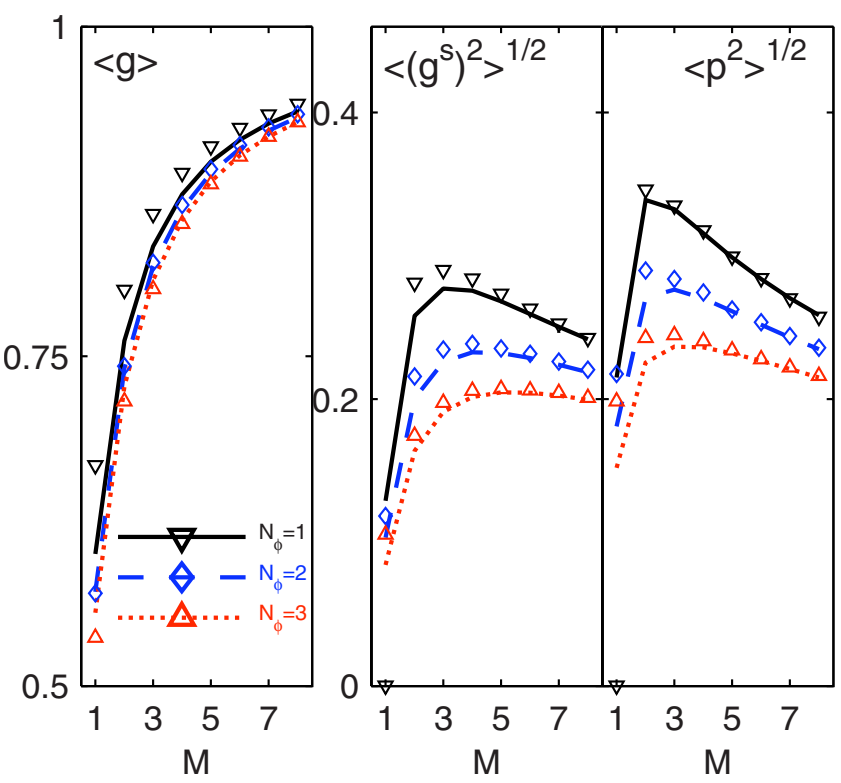

FIG. 4. (Color online) Model with spin-conserving dephasing lead. Numerical (symbols) and analytical (lines) results for normalized mean conductance $\langle g\rangle$, rms spin conductance $g^{s}$, and rms spin polarization $p$ of current exiting a chaotic quantum dot with $N=1$ channel in the entrance lead. $M$ and $N_{\phi}$ are the numbers of channels in the exit and dephasing leads, respectively. An average over $60000 S$ matrices from the CSE was performed for each data point. The lines are from Eqs. (28) and (29).

lead. Averaging these terms separately removes the coherence between the injected spin and the reinjected spin, so they separately average to spinless quantities. As a result, this approximation reproduces the effect of a spin-relaxing dephasing lead. In fact, we find that Eq. (26) matches the exact numerical results for a spin-relaxing lead better than it matches the exact results for a spin-conserving lead, which is consistent with this understanding of the approximations in Eqs. (24) and (25).

Using Eqs. (26) and (18), we find

$$
\left\langle\left(g^{s}\right)^{2}\right\rangle \approx \frac{3 M}{N \Lambda_{\phi}}\left(M-1+N_{\phi} \frac{M^{2}+N(M-1)}{K^{2}}\right),
$$

where $\Lambda_{\phi}=K_{\phi}\left(2 K_{\phi}-1\right)\left(2 K_{\phi}-3\right)$.

Note that Eq. (28) predicts an output spin polarization in all cases with $N_{\phi}>0$, including when there is only one outgoing channel, $M=1$. Numerical results for the spinconserving dephasing lead, using Eq. (23), are shown in Fig. 4 , and they show an rms spin conductance in agreement with Eq. (28) except for $N_{\phi}=M=1$. In the special case of $N_{\phi}$ $=M=1$, an exact treatment shows that $\overrightarrow{g^{s}}=0$, contrary to Eq. (28), even with arbitrary tunnel barriers between the leads and the sample, as shown in Appendix B. Appendix C contains explicit examples of dephasing-induced spin polarization in the case $M=N=1$ in both the spin-conserving and spin-relaxing cases. Numerical results for the spin-relaxing dephasing lead are not shown in Fig. 4, but fall very close to the corresponding lines for the analytic results, even for the 
case $N_{\phi}=M=1$. (A finite spin polarization is allowed in this case, with a dephasing lead that relaxes spin.)

It may seem surprising that dephasing can produce spin polarization in the case $M=1$, where none would be produced in the absence of dephasing. Clearly, the presence of the dephasing lead violates the conditions of the proof in Sec. II that $M=1$ implies $\overrightarrow{g^{s}}=0$, but it is not obvious that dephasing will nonetheless produce polarization, indeed, a single-channel spin-conserving dephasing lead does not, as shown in Appendix B. Nevertheless, we may consider whether the production of spin polarization with $M=1$ is an artifact of the particular third lead dephasing models studied here or a real consequence of inelastic dephasing processes. To further explore this question, we have considered two further variants of the third lead dephasing model.

One could modify the spin-conserving dephasing model to have $N_{\phi}$ dephasing leads each with one channel, each separately reinjecting the same charge/spin that it absorbs. In this model, too, we find that a nonzero $\overrightarrow{g^{s}}$ can be produced for $N_{\phi}>1$ (results not shown).

Brouwer and Beenakker ${ }^{43}$ modified the third lead dephasing model to make dephasing uniform in phase space by placing a tunnel barrier with transparency $\Gamma$ between the dephasing lead and the dot, with $\Gamma \rightarrow 0$ and $N_{\phi} \rightarrow \infty$ while maintaining $\Gamma N_{\phi}=2 \pi \hbar / \Delta \tau_{\phi}$. The $S$ matrix is then not drawn from the CSE, and simple analytical results in the spin-orbit coupled system are challenging. We have studied the spinconserving variant of this model numerically and find that for fixed $\tau_{\phi}$, it gives qualitatively similar results to the simpler model described above; in particular it also gives a nonzero spin current when $M=1$ (results not shown).

All variants of third lead dephasing models considered here show dephasing-induced spin currents with $M=1$. Without a microscopic model of dephasing, however, we cannot rule out the possibility that this effect is an artifact of third lead dephasing models in general. This dephasing-induced polarization is worthy of further study and will be important to consider when designing devices based on the lack of spin polarization with $M=1 .^{44}$

Finally, returning to the single dephasing lead with $\Gamma=1$, we estimate $\left\langle p^{2}\right\rangle \approx\left\langle\left(g^{s}\right)^{2}\right\rangle /\langle g\rangle^{2}$, as in Sec. III, where we modify $\langle g\rangle$ to include the dephasing lead. Using Eqs. (17) and (26), we have

$$
\langle g\rangle \approx \frac{2 M K_{\phi}}{K\left(2 K_{\phi}-1\right)} .
$$

We estimate $\left\langle p^{2}\right\rangle$ using Eqs. (28) and (29). Comparison of these approximations to numerical evaluations is shown in Fig. 4. Again we find that the numerical and analytical results agree qualitatively, except when $N_{\phi}=M=1$.

\section{FINITE BIAS AND TEMPERATURE}

If the source-drain bias $V$ or the temperature $T$ is large enough, the polarization will be further suppressed by electrons of different energy feeling uncorrelated scattering matrices. This effectively increases the number of orbital channels, which decreases the residual polarization. First consider infinitesimal bias at temperature $T$. Adapting Datta, ${ }^{45}$ we take

$$
w^{\text {in }}(\epsilon)=-\frac{\partial f}{\partial \epsilon} \frac{1}{2 N}\left(\begin{array}{ll}
1_{N} & \\
& 0_{M}
\end{array}\right),
$$

where $f(\epsilon)$ is the Fermi distribution. If the scattering matrix for particles of energy $\epsilon$ is $S(\epsilon)$, then $w^{\text {out }}(\epsilon)$ $=S(\epsilon) w^{\text {in }}(\epsilon) S^{\dagger}(\epsilon)$. We approximate $S(\epsilon)$ as correlated only within energy intervals of scale given by the level broadening due to escape into the leads $\Delta^{\prime}=\Delta K / 2$ (see Ref. 46 for an equivalent treatment). That is, we take

$$
\left\langle S_{a b}(\epsilon) S_{c d}^{\dagger}\left(\epsilon^{\prime}\right)\right\rangle=\Delta^{\prime} \delta\left(\epsilon-\epsilon^{\prime}\right)\left\langle S_{a b}(\epsilon) S_{c d}^{\dagger}(\epsilon)\right\rangle,
$$

and

$$
\begin{aligned}
\left\langle S_{a b}(\epsilon)\right. & \left.S_{c d}\left(\epsilon^{\prime}\right) S_{e f}^{\dagger}(\epsilon) S_{g h}^{\dagger}\left(\epsilon^{\prime}\right)\right\rangle \\
= & \left\langle S_{a b}(\epsilon) S_{e f}^{\dagger}(\epsilon)\right\rangle\left\langle S_{c d}\left(\epsilon^{\prime}\right) S_{g h}^{\dagger}\left(\epsilon^{\prime}\right)\right\rangle \\
& +\Delta^{\prime} \delta\left(\epsilon-\epsilon^{\prime}\right)\left\langle S_{a b}(\epsilon) S_{c d}(\epsilon) S_{e f}^{\dagger}(\epsilon) S_{g h}^{\dagger}(\epsilon)\right\rangle,
\end{aligned}
$$

which are valid only for $T \gg \Delta^{\prime}$, which is often true for chaotic quantum dots. For $T \approx \Delta^{\prime},\left\langle S(\epsilon) S^{\dagger}(\epsilon)\right\rangle$ can be calculated using the random Hamiltonian method. ${ }^{47}$

We need an average over a new function,

$$
h\left(\epsilon, \epsilon^{\prime}\right)=f^{\prime}(\epsilon) f^{\prime}\left(\epsilon^{\prime}\right) \operatorname{tr}\left[A S(\epsilon) B S^{\dagger}(\epsilon)\right] \operatorname{tr}\left[C S\left(\epsilon^{\prime}\right) D S^{\dagger}\left(\epsilon^{\prime}\right)\right],
$$

where $A B=A D=C B=C D=0$ and $f^{\prime}=\partial f / \partial \epsilon$. We evaluate the average of $h$ with the $K \times K$ quaternion matrix $S(\epsilon)$ chosen from the CSE along with Eq. (32), giving

$$
\begin{aligned}
\int d \epsilon d \epsilon^{\prime}\left\langle h\left(\epsilon, \epsilon^{\prime}\right)\right\rangle= & \frac{4}{(2 K-1)^{2}} \operatorname{tr} A \operatorname{tr} B \operatorname{tr} C \operatorname{tr} D+\frac{\Delta^{\prime}}{\Lambda} \int d \epsilon f^{\prime}(\epsilon)^{2} \\
& \times\{(K-1)[4 \operatorname{tr} A \operatorname{tr} B \operatorname{tr} C \operatorname{tr} D+\operatorname{tr}(A C) \operatorname{tr}(B D)]-\operatorname{tr} A \operatorname{tr} C \operatorname{tr}(B D)-\operatorname{tr}(A C) \operatorname{tr} B \operatorname{tr} D\} \\
= & \frac{4}{(2 K-1)^{2}} \operatorname{tr} A \operatorname{tr} B \operatorname{tr} C \operatorname{tr} D+\frac{\Delta^{\prime}}{6 T \Lambda} \\
& \times\{(K-1)[4 \operatorname{tr} A \operatorname{tr} B \operatorname{tr} C \operatorname{tr} D+\operatorname{tr}(A C) \operatorname{tr}(B D)]-\operatorname{tr} A \operatorname{tr} C \operatorname{tr}(B D)-\operatorname{tr}(A C) \operatorname{tr} B \operatorname{tr} D\} .
\end{aligned}
$$


Using Eq. (33) in place of Eq. (18), we evaluate $\left\langle\left(g^{s}\right)^{2}\right\rangle$ as above, which simply multiplies Eq. (19) by $\frac{\Delta^{\prime}}{6 T}$. Also, $\langle g\rangle$ is unaffected by temperature, so Eq. (21) is also multiplied by $\Delta^{\prime} / 6 T$.

When dephasing and temperature are both included, the level broadening has a component due to dephasing (effectively due to escape into the dephasing lead) so $\Delta^{\prime}=\Delta\left(K / 2+N_{\phi} / 2\right){ }^{46}$ Equation (28) is then multiplied by $\Delta^{\prime} / 6 T$, and Eq. (29) is unchanged.

If the temperature is small but the source-drain bias $V_{\mathrm{sd}}$ is large compared to $\Delta^{\prime}$, then we can repeat this calculation with

$$
w^{\text {in }}(\epsilon)=\left[\Theta(\epsilon)-\Theta\left(\epsilon-V_{\mathrm{sd}}\right)\right] \frac{1}{2 N V_{\mathrm{sd}}}\left(\begin{array}{cc}
1_{N} & \\
& 0_{M}
\end{array}\right),
$$

where $\Theta$ is the unit step function. Using the equivalent of Eq. (33), this multiplies Eqs. (19), (21), and (28) by $\Delta^{\prime} / V_{\text {sd }}$.

\section{DISCUSSION}

This spin polarization should be able to be produced and detected experimentally. Even quantum dots in $n$-type GaAs/ $\mathrm{AlGaAs}$ heterostructures have been observed to have sufficiently strong spin-orbit coupling to approach the RMT symplectic limit. ${ }^{36,37}$ If the spin-orbit coupling is not strong enough for the $S$ matrices of the dot to be drawn from the CSE, the spin polarization predicted here will be reduced but should still be present. In a given material with fixed spinorbit coupling strength, a sufficiently large quantum dot will be well described by the CSE, with a possible increase in dephasing rate as the dot size increases. Section V shows that the rms spin polarization goes down as $\sqrt{\left(\Delta^{\prime} / V_{\mathrm{sd}}\right)}$ $\propto\left(A V_{\mathrm{sd}}\right)^{-1 / 2}$, so as the dot is made larger to enter the strong spin-orbit limit, the bias range where the results are observable decreases. Thus, the effects predicted in this paper are most likely to be observable in a material with inherently strong spin-orbit coupling, such as $p$-type III/V heterostructures.

If a measurement technique or application is only sensitive to spin polarization along a particular axis, then the rms predictions for the $\mu$ component of the polarization and spin conductance are only $\sqrt{3}$ times smaller than the results stated above, since $\left\langle\left(g_{\mu}^{s}\right)^{2}\right\rangle=\left\langle\left(g^{s}\right)^{2}\right\rangle / 3$ and $\left\langle p_{\mu}^{2}\right\rangle=\left\langle p^{2}\right\rangle / 3$.

We have shown that quantum dots with spin-orbit coupling can generate spin-polarized currents without magnetic fields or ferromagnets, except in the case of only one outgoing channel, when such a device can only produce a spin current if dephasing is present. These mesoscopic fluctuations can be large enough to give appreciable spin currents in devices with a small number of propagating channels. Even if the spin-orbit coupling is weak, a sufficiently large device will show these effects. Dephasing generally decreases the spin polarization, except in the case of one outgoing channel, where spin polarization cannot be produced in the absence of dephasing.

\section{ACKNOWLEDGMENTS}

We acknowledge helpful discussions with Caio Lewenkopf, Emmanuel Rashba, Ilya Finkler, Philippe Jacquod, and particularly Ari Turner, who suggested the method of choosing matrices from the CSE. We note the use of the Quaternion Toolbox for MATLAB, created by S. J. Sangwine and N. Le Bihan. This work was supported in part by the Fannie and John Hertz Foundation and NSF under Grants No. PHY0646094 and No. DMR-0541988.

\section{APPENDIX A: QUATERNIONS}

A quaternion $q$ is a $2 \times 2$ matrix of complex numbers,

$$
q=q^{(0)} \mathbb{1}_{2}+i \sum_{\mu=1}^{3} q^{(\mu)} \sigma_{\mu},
$$

where $\sigma_{\mu}$ are the Pauli matrices and $q^{(\mu)} \in \mathrm{C}$. We define three conjugates of $q$,

$$
\begin{gathered}
\text { complex conjugate: } q^{*}=q^{(0) *} 1_{2}+i \sum q^{(\mu) *} \sigma_{\mu}, \\
\text { quaternion dual: } q^{R}=q^{(0)} \rrbracket_{2}-i \sum q^{(\mu)} \sigma_{\mu}, \\
\text { Hermitian conjugate: } \quad q^{\dagger}=q^{R *} .
\end{gathered}
$$

If the $2 \times 2$ matrix

$$
\left(\begin{array}{ll}
a & b \\
c & d
\end{array}\right)
$$

is expressed as a quaternion $q$, then in the $2 \times 2$ notation for the quaternion,

$$
\begin{aligned}
q^{*} & =\left(\begin{array}{cc}
d^{*} & -c^{*} \\
-b^{*} & a^{*}
\end{array}\right), \\
q^{R} & =\left(\begin{array}{cc}
d & -b \\
-c & a
\end{array}\right), \\
q^{\dagger} & =\left(\begin{array}{ll}
a^{*} & c^{*} \\
b^{*} & d^{*}
\end{array}\right) .
\end{aligned}
$$

It is clear that $q^{\dagger}$ corresponds to the usual definition of Hermitian conjugation, but complex conjugation is not equivalent.

For a $K \times K$ matrix of quaternions $Q$ we similarly define

$$
\begin{aligned}
& \left(Q^{*}\right)_{i j}=Q_{i j}^{*}, \\
& \left(Q^{R}\right)_{i j}=Q_{j i}^{R}, \\
& \left(Q^{\dagger}\right)=\left(Q^{*}\right)^{R},
\end{aligned}
$$

where again Hermitian conjugation of a $K \times K$ quaternion matrix corresponds to the usual Hermitian conjugation of the equivalent $2 K \times 2 K$ complex matrix, but complex conjugation is not equivalent. By convention, the trace of the quaternion matrix $Q$ is 


$$
\operatorname{tr} Q=\sum_{i} Q_{i i}^{(0)},
$$

which accords with the usual definition of the trace of the equivalent complex matrix (since the Pauli matrices have zero trace), except that the quaternion trace is a factor of 2 smaller.

\section{APPENDIX B: SPIN-POLARIZATION FORBIDDEN WITH $M=N_{\phi}=1$}

Consider a quantum dot with $N$ input channels, one output channel, and one spin-conserving dephasing channel. We show here that if we send an unpolarized incoherent current in the $N$ channels and measure the spin polarization in the output channel, then as long as TRS is unbroken, there can be no spin polarization in the measured channel, independent of the transparency of the contacts.

From Eq. (23), we have $w^{\text {in }}=w_{0}^{\text {in }}+w_{\phi}^{\text {in }}$ where

$$
w_{\phi}^{\text {in }}=P_{\phi} \sigma_{\mu} \operatorname{tr}\left(\sigma_{\nu} P_{\phi} S w_{0}^{\text {in }} S^{\dagger}\right)\left(N_{\phi} \delta_{\mu \nu}-\Xi_{\mu \nu}\right)^{-1},
$$

and $g_{\mu}^{s}=2 \operatorname{tr}\left(\sigma_{\mu} P_{K} w^{\text {out }}\right)=2\left[w_{K K}^{\text {out }}\right]^{(\mu)}$, where $P_{K}$ is the projection onto the $K$ th channel (the output channel) and $[q]^{(\mu)}$ is the $\mu$ component of the quaternion $q$.

The outgoing density matrix is $w^{\text {out }}=w_{0}^{\text {out }}+w_{\phi}^{\text {out }}$, where $w_{0}^{\text {out }}=S w_{0}^{\text {in }} S^{\dagger}$ and $w_{\phi}^{\text {out }}=S w_{\phi}^{\text {in }} S^{\dagger}$. For convenience of notation, the outgoing channel has index $K$ and the dephasing lead channel has index 1 , so $P_{\phi}=P_{1}$. Now

$$
w_{0}^{\text {in }}=\frac{1}{2 N}\left(1-P_{K}-P_{1}\right),
$$

SO

$$
\left(w_{0}^{\text {out }}\right)_{K K}=\frac{1}{2 N}\left(1-S_{K K} S_{K K}^{*}-S_{K 1} S_{1 K}^{*}\right) .
$$

As in Sec. II, the first two terms are real, but the third term can have a quaternion component. We show that $S_{K 1} S_{1 K}^{*}$ is exactly canceled by $\left(w_{\phi}^{\text {out }}\right)_{K K}$ in the final result for $w^{\text {out }}$. We have

$$
\operatorname{tr}\left(\sigma_{\nu} P_{1} S w_{0}^{\mathrm{in}} S^{\dagger}\right)=\left[\frac{\sigma_{\nu}}{2 N}\left(1-S_{1 K} S_{K 1}^{*}-S_{11} S_{11}^{*}\right)\right]^{(0)}
$$

and

$$
\Xi_{\mu \nu}=\operatorname{tr}\left(\sigma_{\nu} P_{1} S \sigma_{\mu} P_{1} S^{\dagger}\right)=\delta_{\mu \nu} S_{11} S_{11}^{*},
$$

where the last equality follows because $S_{11}$ commutes with $\sigma_{\mu}$, since $S_{11} \in \mathrm{C}$. Together, these give

$$
w_{\phi}^{\text {in }}=P_{1} \sigma_{\mu} \frac{\left[\frac{\sigma_{\mu}}{2 N}\left(1-S_{1 K} S_{K 1}^{*}-S_{11} S_{11}^{*}\right)\right]^{(0)}}{1-S_{11} S_{11}^{*}},
$$

with implied summation over $\mu$. For $q$ a quaternion, $\sigma_{\mu}\left[\sigma_{\mu} q\right]^{(0)}=q$, so

$$
w_{\phi}^{\text {in }}=\frac{P_{1}}{2 N}\left(1-\frac{S_{1 K} S_{K 1}^{*}}{1-S_{11} S_{11}^{*}}\right) .
$$

Then

$$
w_{\phi K K}^{\text {out }}=\frac{1}{2 N}\left(S_{K 1} S_{1 K}^{*}-\frac{S_{K 1} S_{1 K} S_{K 1}^{*} S_{1 K}^{*}}{1-S_{11} S_{11}^{*}}\right) .
$$

The first term in Eq. (B8) cancels $S_{K 1} S_{1 K}^{*}$ in Eq. (B3). The second term is real, since $S=S^{R}$, so $S_{K 1} S_{1 K}=S_{K 1} S_{K 1}^{R} \in \mathbb{C}$. Thus, $w_{K K}^{\text {out }}$ is real and $\overrightarrow{g^{s}}=0$. Since this result relies only on the unitarity and self-duality of the $S$ matrix, it is true independent of the transparency of the dephasing contact. Note, however, that if $N_{\phi}>1$, then $\overrightarrow{g^{s}}$ can be nonzero in this theory, even when $M=1$.

\section{APPENDIX C: SPIN POLARIZATION FROM DEPHASING}

Here we give explicit examples of dephasing-induced spin polarization in the case $M=N=1$ for both the spinconserving and spin-relaxing dephasing leads. The key to the operation of the dephasing leads is that they break coherence between different spin states. The spin-relaxing dephasing lead breaks the coherence between the different spins within a single channel and can thus produce a spin conductance with $N_{\phi}=1$. The spin-conserving dephasing lead does not break coherence between modes within a single channel and thus requires $N_{\phi}=2$ to produce a spin polarization.

In the case of a spin-relaxing dephasing lead, consider an example of an $S$ matrix in the complex representation with $M=N=N_{\phi}=1$,

$$
S=\left(\begin{array}{cccccc}
0 & 0 & 0 & 0 & \alpha & -\alpha \\
0 & 0 & 0 & 1 & 0 & 0 \\
1 & 0 & 0 & 0 & 0 & 0 \\
0 & 0 & 0 & 0 & \alpha & \alpha \\
0 & \alpha & \alpha & 0 & 0 & 0 \\
0 & \alpha & -\alpha & 0 & 0 & 0
\end{array}\right),
$$

where $\alpha=1 / \sqrt{2}$. An up-spin incident from the left exits as an up spin to the right. A down-spin incident from the left enters the dephasing lead as an $x$-polarized spin. It is reinjected as a dephased equal combination of up- and down-polarized spins, which then exit $50 \%$ as up spins to the left and $50 \%$ as down spins to the right. This results in $g=3 / 4, \overrightarrow{g^{s}}=\hat{z} / 4$, and $\vec{p}=\hat{z} / 3$.

We can obtain this result formally, applying Eq. (27) to find $w_{\phi}^{\text {in }}=P_{\phi} / 4$. Note that we divided it by 2 because we are using the complex rather than quaternion representation. Applying Eq. (4), $\overrightarrow{g^{s}}=\hat{z} / 4$. Note that if the dephasing lead had preserved the spin of its absorbed current, it would have reinjected $x$-polarized spins, which would have all exited as spin down to the right, giving $g=1$ and $\overrightarrow{g^{s}}=0$, as Appendix B proves must happen. 
For the spin-conserving dephasing lead, consider a quantum dot with $M=1=N$ and $N_{\phi}=2$ with complex $S$ matrix,

$$
S=\left(\begin{array}{cccccccc}
0 & 0 & 0 & 0 & 0 & -\alpha & 0 & \alpha \\
0 & 0 & 0 & 1 & 0 & 0 & 0 & 0 \\
1 & 0 & 0 & 0 & 0 & 0 & 0 & 0 \\
0 & 0 & 0 & 0 & 0 & \alpha & 0 & \alpha \\
0 & \alpha & \alpha & 0 & 0 & 0 & 0 & 0 \\
0 & 0 & 0 & 0 & 0 & 0 & -1 & 0 \\
0 & -\alpha & \alpha & 0 & 0 & 0 & 0 & 0 \\
0 & 0 & 0 & 0 & 1 & 0 & 0 & 0
\end{array}\right) .
$$

Again, an up-spin incident from the left exits as an up spin to the right. A down-spin incident from the left enters the dephasing lead as a superposition of spin up in mode 1 and mode 2 . This spin is conserved but the phase between the modes is broken. After reinjection, the current reflects back into the dephasing lead, this time as a superposition of spin down in both modes. Again the spin is conserved but dephased, and it exits as equal parts spin up to the left and spin down to the right, which again gives $g=3 / 4, \overrightarrow{g^{s}}=\hat{z} / 4$, and $\vec{p}=\hat{z} / 3$. Using Eq. (23), $w_{\phi}^{\text {in }}=P_{\phi} / 4$, just as in the previous example.
${ }^{1}$ H.-A. Engel, E. I. Rashba, and B. I. Halperin, in Handbook of Magnetism and Advanced Magnetic Materials, edited by $\mathrm{H}$. Kronmüller and S. Parkin (Wiley, Chichester, UK, 2007), Vol. 5, pp. 2858-2877.

${ }^{2}$ L. S. Levitov, Y. V. Nazarov, and G. M. Éliashberg, Sov. Phys. JETP 61, 133 (1985).

${ }^{3}$ V. M. Edelstein, Solid State Commun. 73, 233 (1990).

${ }^{4}$ A. G. Aronov, Y. B. Lyanda-Geller, and G. E. Pikus, Sov. Phys. JETP 73, 537 (1991).

${ }^{5}$ M. Trushin and J. Schliemann, Phys. Rev. B 75, 155323 (2007).

${ }^{6}$ Z. Huang and L. Hu, Phys. Rev. B 73, 113312 (2006).

${ }^{7}$ Y. A. Bychkov and E. I. Rashba, J. Phys. C 17, 6039 (1984).

${ }^{8}$ S. Chesi and G. F. Giuliani, arXiv:cond-mat/0701415 (unpublished).

${ }^{9}$ A. V. Chaplik, M. V. Entin, and L. I. Magarill, Physica E (Amsterdam) 13, 744 (2002).

${ }^{10}$ J.-F. Liu, Z.-C. Zhong, L. Chen, D. Li, C. Zhang, and Z. Ma, Phys. Rev. B 76, 195304 (2007).

${ }^{11}$ Y. Jiang and L. Hu, Phys. Rev. B 74, 075302 (2006).

${ }^{12}$ Y. Xing, Q. Sun, L. Tang, and J. Hu, Phys. Rev. B 74, 155313 (2006).

${ }^{13}$ Y. K. Kato, R. C. Myers, A. C. Gossard, and D. D. Awschalom, Phys. Rev. Lett. 93, 176601 (2004).

${ }^{14}$ S. Ganichev, S. Danilov, P. Schneider, V. Belkov, L. Golub, W. Wegscheider, D. Weiss, and W. Prettl, J. Magn. Magn. Mater. 300, 127 (2006).

${ }^{15}$ A. Y. Silov, P. A. Blajnov, J. H. Wolter, R. Hey, K. H. Ploog, and N. S. Averkiev, Appl. Phys. Lett. 85, 5929 (2004).

${ }^{16}$ A. A. Kiselev and K. W. Kim, Appl. Phys. Lett. 78, 775 (2001).

${ }^{17}$ M. Governale and U. Zülicke, Phys. Rev. B 66, 073311 (2002).

${ }^{18}$ P. G. Silvestrov and E. G. Mishchenko, Phys. Rev. B 74, 165301 (2006).

${ }^{19}$ M. Eto, T. Hayashi, and Y. Kurotani, J. Phys. Soc. Jpn. 74, 1934 (2005).

${ }^{20}$ Z.-F. Jiang, J. Li, S.-Q. Shen, and W. M. Liu, arXiv:0709.1566 (unpublished).

${ }^{21}$ M. Scheid, A. Pfund, D. Bercioux, and K. Richter, Phys. Rev. B 76, 195303 (2007).

${ }^{22}$ A. A. Kovalev, L. P. Zrbo, Y. Tserkovnyak, G. E. W. Bauer, and J. Sinova, arXiv:0711.4430 (unpublished).
${ }^{23}$ A. Mal'shukov and C. S. Chu, arXiv:0712.0442 (unpublished).

${ }^{24}$ M. Duckheim and D. Loss, arXiv:0805.4143 (unpublished).

${ }^{25}$ F. Zhai and H. Q. Xu, Phys. Rev. Lett. 94, 246601 (2005).

${ }^{26}$ A. A. Kiselev and K. W. Kim, Phys. Rev. B 71, 153315 (2005).

${ }^{27}$ J. J. Krich and B. I. Halperin (unpublished).

${ }^{28}$ J. H. Bardarson, I. Adagideli, and P. Jacquod, Phys. Rev. Lett. 98, 196601 (2007).

${ }^{29}$ M. L. Mehta, Random Matrices (Elsevier, New York, 2004).

${ }^{30}$ M. Büttiker, Y. Imry, R. Landauer, and S. Pinhas, Phys. Rev. B 31, 6207 (1985).

${ }^{31}$ B. K. Nikolić and S. Souma, Phys. Rev. B 71, 195328 (2005).

${ }^{32}$ Y. Jiang and L. Hu, Phys. Rev. B 75, 195343 (2007).

${ }^{33}$ C. A. Perroni, D. Bercioux, V. M. Ramaglia, and V. Cataudella, J. Phys.: Condens. Matter 19, 186227 (2007).

${ }^{34}$ P. Sharma and P. W. Brouwer, Phys. Rev. Lett. 91, 166801 (2003).

${ }^{35}$ C. W. J. Beenakker, Rev. Mod. Phys. 69, 731 (1997).

${ }^{36}$ D. M. Zumbühl, J. B. Miller, C. M. Marcus, K. Campman, and A. C. Gossard, Phys. Rev. Lett. 89, 276803 (2002).

${ }^{37}$ D. M. Zumbühl, J. B. Miller, C. M. Marcus, D. GoldhaberGordon, J. J. S. Harris, K. Campman, and A. C. Gossard, Phys. Rev. B 72, 081305(R) (2005).

${ }^{38}$ P. W. Brouwer and C. W. J. Beenakker, J. Math. Phys. 37, 4904 (1996).

${ }^{39}$ P. Forrester, Log-Gases and Random Matrices (unpublished), Chap. 2, www.ms.unimelb.edu.au/ matpjf/b2.ps

${ }^{40}$ M. Büttiker, IBM J. Res. Dev. 32, 63 (1988).

${ }^{41}$ P. W. Brouwer and C. W. J. Beenakker, Phys. Rev. B 51, 7739 (1995)

${ }^{42}$ H. U. Baranger and P. A. Mello, Phys. Rev. B 51, 4703 (1995).

${ }^{43}$ P. W. Brouwer and C. W. J. Beenakker, Phys. Rev. B 55, 4695 (1997).

${ }^{44}$ F. Zhai, K. Chang, and H. Q. Xu, Appl. Phys. Lett. 92, 102111 (2008).

${ }^{45}$ S. Datta, Electronic Transport in Mesoscopic Systems (Cambridge University Press, Cambridge, England, 1995).

${ }^{46}$ A. G. Huibers, S. R. Patel, C. M. Marcus, P. W. Brouwer, C. I. Duruöz, and J. S. Harris, Phys. Rev. Lett. 81, 1917 (1998).

${ }^{47}$ J. J. M. Verbaarschot, H. A. Weidenmuller, and M. R. Zirnbauer, Phys. Rep. 129, 367 (1985). 